\title{
Dispersion Estimation From Linear Array Data in the Time-Frequency Plane
}

\author{
Antoine Roueff, Jérôme I. Mars, Jocelyn Chanussot, and Helle Pedersen
}

\begin{abstract}
We consider the problem of estimating the dispersion of a wave field from data recorded by a linear array of geophones. The fact that the data we are looking at may contain several propagating waves make this even more challenging. In this paper, a new algorithm is proposed to solve this issue. Currently, there are two methods for estimating wave dispersion described in the literature. The first method estimates the group delay function from the time-frequency representation (TFR) of each sensor separately. It is efficient as long as the patterns of the different waves do not overlap in the time-frequency plane. The second method estimates the dispersion from the two-dimensional (2-D) Fourier transform of the profile (or more generally from a velocity-frequency representation). This assumes that the dispersion is constant along the entire sensor array. It is efficient as long as the patterns of the waves do not overlap in the frequency domain. Our method can be thought of as a hybrid of the above two methods as it is based on the construction of a TFR where the energy of waves that propagate at a selected velocity are amplified. The primary advantage of our algorithm is the use of the velocity variable to separate the patterns of the propagating waves in the time-frequency plane. When applied to both synthetic and real data, this new algorithm gives much improved results when compared with other standard methods.
\end{abstract}

Index Terms-Dispersion, linear array, radon transform, timefrequency representations.

\section{INTRODUCTION}

A NALYSIS of seismic surface waves applied to near surface structures is an increasingly important tool in civil and seismic engineering [1]. Surface waves are highly energetic and are dispersive because their depth penetration depends on their wavelength (i.e., on the frequency). This implies that the different harmonics of the wave propagate at different velocities, and consequently, the wave's duration (in the time domain) tends to increase with distance. Within the signal processing community, these waves have been widely studied. Their description in the time-frequency plane is problematic because their dispersive characteristics make the standard quadratic time-frequency representations (TFRs) (such as the Wigner distribution) unsuitable [2], [3]. In this paper, we consider data

Manuscript received February 13, 2004; revised November 25, 2004. The associate editor coordinating the review of this manuscript and approving it for publication was Prof. Fredrik Gustafsson.

A. Roueff is with the Commissariat à l'Energie Atomique CEA/DASE/ LDG/DSO, 91680 Bruyères-le-Chatel, France (e-mail: antoine.roueff@cea.fr).

J. I. Mars and J. Chanussot are with the Laboratoire des Images et des Signaux (LIS), CNRS, UMR 5083, OSUG, GDR, Information Signal Image viSion (ISIS), Saint Martin d'Hères Cédex, France (e-mail: jerome.mars@lis.inpg.fr; jocelyn.chanussot@lis.inpg.fr).

H. Pedersen is with the Laboratoire de Géophysique Interne et de Tectonophysique (LGIT), Grenoble, France (e-mail: Helle.Pedersen@obs.ujfgrenoble.fr).

Digital Object Identifier 10.1109/TSP.2005.855430 recorded by a linear array of sensors. Our approach is somewhat different to that of the classical time-frequency literature as we want to construct a TFR from a multisignal analysis.

We show an example of a dispersive wave in Fig. 3(a). This synthetic profile ${ }^{1}$ of a dispersive wave has a horizontal axis referencing the sample number and a vertical axis showing the sensor number. In our case, where the array is linear, this vertical axis can be thought of as a distance variable corresponding to the distance between the source and the sensor. From the figure, one can see that the filter that models the propagation is not a simple delay; there is phase distortion of the wave. In this paper, we focus on the estimation of the dispersion of surface waves from a seismic profile. The fact that we consider data containing several wave fields adds to the difficulty of this problem. Actually, when estimating the dispersion of one wave, the noise part of the data that disturb this processing is the random noise and the other waves that are also present in the data.

This analysis has two applications. First, in civil engineering, the parameters of the dispersive waves make it possible to invert for the shear velocity as a function of depth. Second, in petroleum exploration, energetic surface waves are often considered as noise, and the relevant signal part of the data corresponds to volume waves. Consequently, the rejection of the surface waves (simplified by dispersion estimation) greatly improves the signal-to-noise ratio (SNR) [4], [5].

Two groups of methods are proposed in the literature for the estimation of dispersion. The first group is based on an improved interpretation of the TFRs for each trace separately [6]-[8]. These one-dimensional (1-D) methods lead to the estimation of the group velocity between the source and each sensor. The second set of methods is a multisensor analysis that leads to a global (or mean) estimation of the phase velocity between the sensors using velocity-frequency representations [9], [10]. The advantages and drawbacks of those two classical approaches are reviewed in Section II. As explained in Section III, our method takes advantage of the classical ones by designing a multisensor analysis in the time-frequency plane. Section IV is devoted to the application of the different methods to real data.

\section{Classical Methods of DisPersion Estimation}

\section{A. One-Dimensional Analysis}

The first set of classical methods to be discussed here is based on single sensor analysis (1-D analysis). To illustrate these methods, we consider a signal $s$ containing a synthetic wave $w$ recorded by a sensor at the distance $D$ from the source.

\footnotetext{
${ }^{1} \mathrm{~A}$ profile is the image formed by the stacking of the different recorded signals.
} 


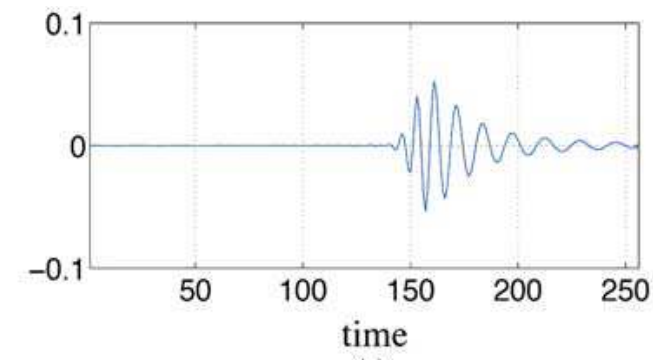

(a)

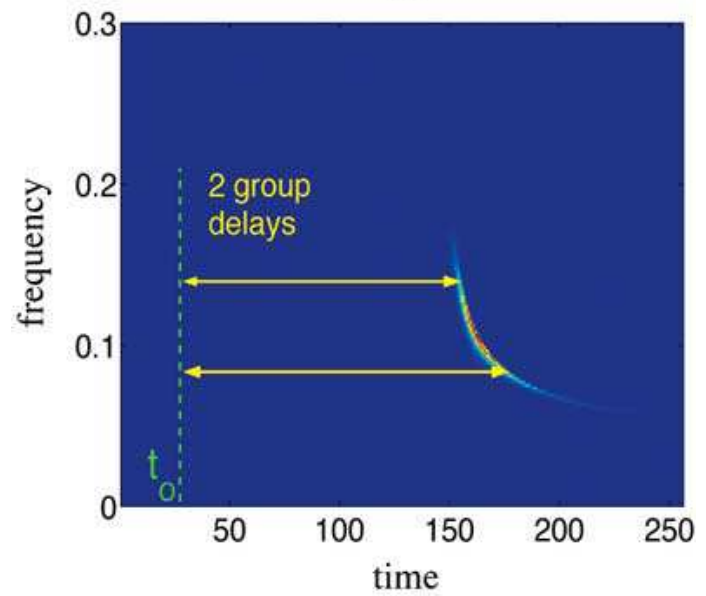

(b)

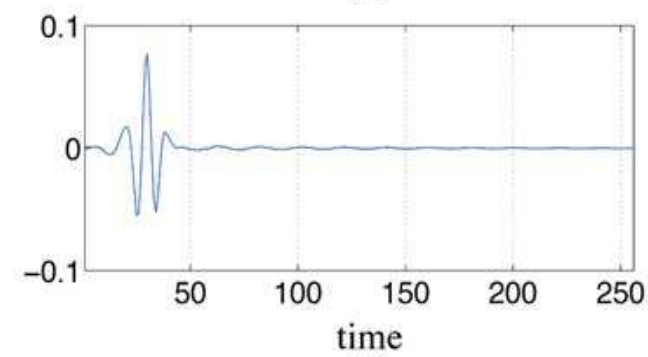

(c)

Fig. 1. Estimation and correction of dispersion using a reassigned spectrogram. (a) Initial record. (b) Reassigned Spectrogram. (c) Record after dispersion correction.

The time representation of such a signal is shown in Fig. 1(a). The transfer function between the source and the sensor is denoted by $h$. This method assumes that there is no attenuation during the propagation, so the modulus of the Fourier transform (FT) of $\mathrm{h}$ [denoted by $H(\nu)]$ is equal to 1

$$
H(\nu)=e^{i \phi_{H}(\nu)}
$$

where $\phi_{H}(\nu)$ is the phase of $H(\nu)$, (i.e., $\phi_{H}(\nu)=\angle H(\nu)$ ).

When it is assumed that, at the source, all the harmonics of the wave are localized at the same instant $t_{o}$, the transfer function $H(\nu)$ can be written as

$$
H(\nu)=e^{i\left(-2 \pi \int_{0}^{\nu}\left(\tau_{g}(f)-t_{o}\right) d f+\phi_{H}(0)\right)}
$$

where $\tau_{g}(f)$ is the group delay of the wave at frequency $f$ as it is defined in the signal processing community, and $\tau_{g}(f)-t_{o}$ is the group delay of the wave between the source and the sensor at

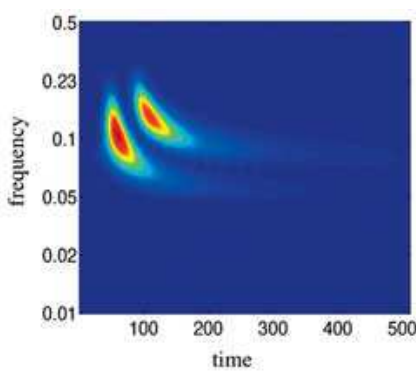

(a)

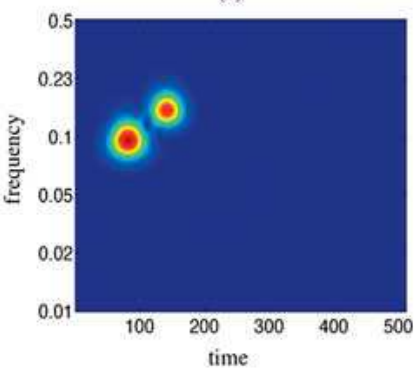

(c)

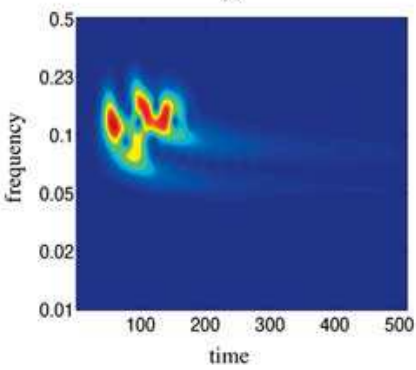

(e)

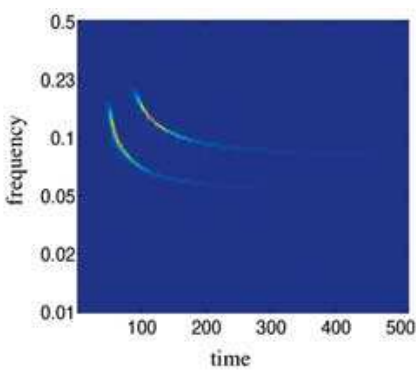

(b)

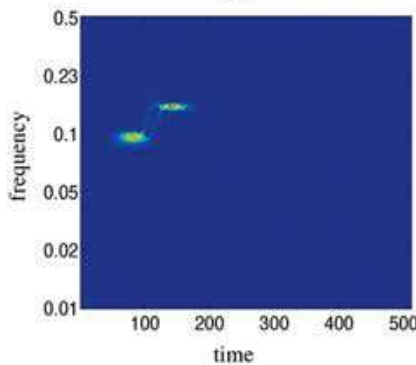

(d)

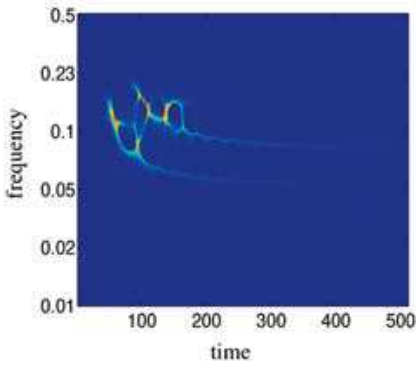

(f)
Fig. 2. Examples of issues of overlap for ridge estimation of several waves. (a) Signal 1 (scalogram). (b) Signal 1 (reassigned scalogram). (c) Signal 2 (scalogram). (d) Signal 2 (reassigned scalogram). (e) Signal 1+2 (scalogram). (f) Signal 1+2 (reassigned scalograms).

frequency $f$. This delay is directly related to the group velocity $v_{g}(f)$ by

$$
v_{g}(f)=\frac{D}{\tau_{g}(f)-t_{o}} .
$$

To estimate the dispersion, we need to calculate $H$. From (2), the unknown function to be estimated is $\tau_{g}(f)$. Different methods are available to estimate $\tau_{g}(f)$ depending on the TFR used [11]-[13]. In this paper, we use the ridge of the reassigned spectrogram (or scalogram) [14] as proposed by Pedersen et al. in [8]. The delay $\tau_{g}(f)-t_{o}$ at each frequency is estimated as the duration between the origin $t_{o}$ and the ridge of the pattern of the wave, as illustrated in Fig. 1(b). This method estimates efficiently $\phi_{H}$. However, when there is no a priori information, two parameters, namely, the phase shift $\phi_{H}(0)$ and the delay $t_{o}$ from (2), are unknown and are arbitrarily fixed.

Once $H$ is estimated, it is possible to compensate for the propagation of the wave. Fig. 1(c) shows the signal $s$, filtered by $H^{-1}$, which is concentrated around $t=t_{o}$. The major issue with this 1-D technique is that in the presence of several waves with close time-frequency locations, there may be some overlapping phenomena between the patterns of the different waves. As a consequence, the ridges of the different components may be impossible to characterize. This is illustrated in Fig. 2, where 


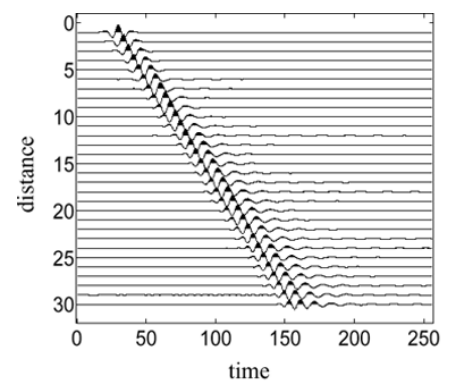

(a)

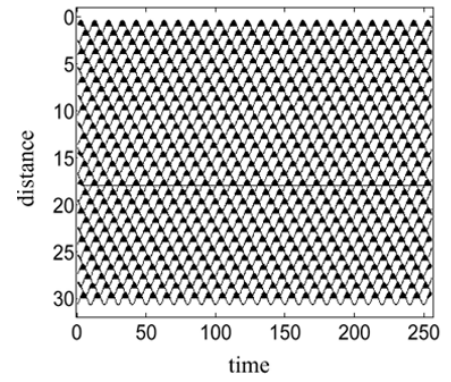

(c)

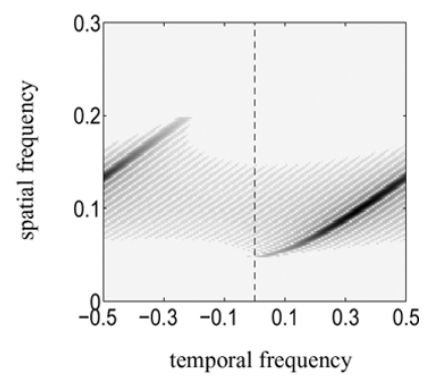

(b)

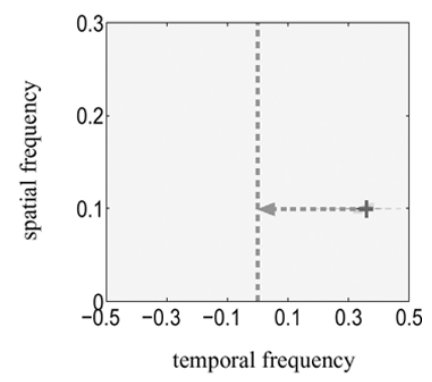

(d)
Fig. 3. Profile filtered at frequency $f_{t}$. (a) Initial profile. (b) $|2 D F T|$ of (a). (c) Filtered profile by a complex exponential. (d) $|2 D F T|$ of (c).

both scalograms and reassigned scalograms are shown. In (a) and (b) or (d) and (e), the ridges can easily be defined, but in (c) and (f), the patterns overlap, making interpretation impossible.

Note that in this paper, we intend to compare different dispersion estimation methods from a seismic profile whose dispersion is assumed to be spatially stationary (i.e., constant all along the sensor array). The application of this 1-D method to a profile is performed by estimating the dispersion at all the individual sensors, and afterwards, a global result is found by taking the mean of the different estimations [7]. This method is suitable when only a few sensors are available.

\section{B. Multisensor Analysis}

The second set of classical methods presented is based on multisensor analysis. To introduce them, we study a synthetic dispersive wave [refer to the time domain Fig. 3(a)]. In Fig. 3(b), we show the modulus of the two dimensional Fourier transform (2DFT, which is also called the $f-k$ spectrum $^{2}$ ) of the profile [4]. The temporal and spatial frequencies are, respectively, related to the time and distance (number of sensor) variables. Note that in the frequency domain, the space variable is no longer on the vertical axis but on the horizontal one. The $f-k$ spectrum is typically represented in this way by the geophysical community. All axes use normalized frequencies, and within the $f-k$ images, we focus on the 0 to 0.3 temporal frequencies.

As we will show later, the nonlinearity of the pattern gives evidence of the wave's dispersion. The dispersion between sensor $n$ and sensor $n+1$ is characterized by the transfer function denoted $h_{n}$. Denoting $w_{n}$ as the dispersive wave at sensor $n$, we get $w_{n+1}=w_{n} * h_{n}$, where $*$ stands for the convolution operator. $H_{n}(f)$ the Fourier transform $(\mathrm{FT})$ of $h_{n}$ can be

\footnotetext{
${ }^{2}$ In the name $f-k$ spectrum, the $f$ refers to temporal frequency and the $k$ to the wave number linked to the spatial frequency.
}

written $H_{n}(f)=\left|H_{n}(f)\right| e^{j \phi_{H_{n}}(f)}$, where $\phi_{H_{n}}(f)$ is the phase of $H_{n}(f)$. As stated in the previous paragraph, the dispersion is assumed to be spatially stationary along all the $N$ sensors (i.e., $h_{n}=h$ ), and the modulus $|H(f)|$ is assumed to be equal to one (no attenuation). Thus, $H(f)=e^{j \phi_{H}(f)}$. The phase velocity $v_{\phi}$ is directly related to $\phi_{H}(f)$ by

$$
v_{\phi}(f)=\frac{2 \pi f d}{\phi_{H}(f)}
$$

where $d$ is the distance between two sensors.

In presence of one dispersive wave, the $n$th trace is equal to the original wave $w$ convolved $n$ times with $h$ :

$$
p(n, t)=\underbrace{h *(h *(\ldots *(h * w))) .}_{n \text { times }}
$$

Hence, the profile $p$ in the time-space domain can be modeled by

$$
p(n, t)=F T_{t}^{-1}\left[W(f) \cdot e^{j n \phi_{H}(f)}\right](t)
$$

where $n=0,1, \ldots N-1$ is the sensor number, $t$ is time, and $W(f)$ is the temporal Fourier transform $\left(\mathrm{FT}_{t}\right)$ of $w(t)$.

The estimation of $\phi_{H}\left(f_{t}\right)$ for a given temporal frequency $f_{t}$ using classical multisensor methods is two-step process. First, the profile at frequency $f_{t}$ is analyzed by computing the temporal Fourier transform at $f_{t}$. In time, the filtered profile denoted by $p_{f_{t}}$ becomes

$$
p_{f_{t}}(n, t)=F T_{t}^{-1}\left[W\left(f_{t}\right) \cdot e^{j n \phi_{H}\left(f_{t}\right)} \delta\left(f-f_{t}\right)\right]
$$

which is equal to

$$
p_{f_{t}}(n, t)=W\left(f_{t}\right) \cdot e^{j\left(n \phi_{H}\left(f_{t}\right)+2 \pi t f_{t}\right)} .
$$

This results in the profile shown in Fig. 3(c) in the time domain (real part) and in Fig. 3(d) in $f-k$ space. The second step in the estimation of $\phi_{H}\left(f_{t}\right)$ is to find the correction that aligns the sinusoids in Fig. 3(c). This second operation actually estimates the correction that compensates for the dispersion at $f=f_{t}$. For this particular correction, the delay between one sensor and the next is null. Thus, in the frequency domain, this corresponds to the case where the point is located at the zero value on the spatial frequency axes (vertical) in Fig. 3(d) (see the arrow). In practice, two corrections are used to align the sine waves. The first one is a phase shift of the profile. More precisely, it corresponds to the application of a phase shift $n \phi$ to each trace $n$ (of the profile). The second one is a delay of the profile. It corresponds to the application of the delay $n \tau$ to each trace $n$.

In both cases, the criterion to find the best correction is the maximization of the energy of the signal corresponding to the summation of all traces (after correction). In the first case, the signal corresponding to the summation of all traces is

$$
S\left(t, f_{t}, \phi\right)=\sum_{n=0}^{N-1} p_{f_{t}}(n, t) e^{j n \phi}
$$

which leads to

$$
S\left(t, f_{t}, \phi\right)=\sum_{n=0}^{N-1} W\left(f_{t}\right) e^{j\left(n\left(\phi_{H}\left(f_{t}\right)+\phi\right)+2 \pi t f_{t}\right)}
$$


and then to

$$
S\left(t, f_{t}, \phi\right)=W\left(f_{t}\right) e^{2 \pi t f_{t}} \sum_{n=0}^{N-1} e^{j n\left(\phi_{H}\left(f_{t}\right)+\phi\right)}
$$

Thus, the phase shift $\hat{\phi}\left(f_{t}\right)$ that maximizes the energy of $S\left(t, f_{t}, \phi\right)$ is

$$
\hat{\phi}\left(f_{t}\right)=\arg \left(\max _{\phi}\left|W\left(f_{t}\right) e^{2 \pi t f_{t}} \sum_{n=0}^{N-1} e^{j n\left(\phi_{H}\left(f_{t}\right)+\phi\right)}\right|\right) .
$$

This is equivalent to

$$
\hat{\phi}\left(f_{t}\right)=\arg \left(\max _{\phi}\left|\sum_{n=0}^{N-1} e^{j n\left(\phi_{H}\left(f_{t}\right)+\phi\right)}\right|\right) .
$$

This leads to

$$
\hat{\phi}\left(f_{t}\right)=-\phi_{H}\left(f_{t}\right)[2 \pi]
$$

where $[2 \pi]$ stands for modulo $2 \pi$.

Replacing $\phi$ by $-2 \pi f_{n}$ and applying a similar procedure to all $f_{t}$ s leads to a two-dimensional (2-D) representation $\left(f_{t}, f_{n}\right)$ that is actually the 2-D Fourier transform (2DFT) of $p(t, n)$ [9]. Its modulus is shown in Fig. 3(b), where one can visualize $\phi_{H}\left(f_{t}\right)=2 \pi \hat{f}_{n}\left(f_{t}\right)[2 \pi]$ at the point of maximum energy in each line (each frequency). Note that the modulo $2 \pi$ results in an aliasing effect in the $f-k$ pattern, which can hinder our understanding of this frequency representation. For this reason, the pattern is split into two parts.

In the second case, we apply a delay correction $\tau$ to the profile. Since the calculation is similar, the signal corresponding to the summation of all traces is given by

$$
S\left(t, f_{t}, \tau\right)=\sum_{n=0}^{N-1} p_{f_{t}}(n, t+n \tau)
$$

which leads to

$$
S\left(t, f_{t}, \tau\right)=W\left(f_{t}\right) e^{2 \pi t f_{t}} \sum_{n=0}^{N-1} e^{j n\left(2 \pi \tau f_{t}+\phi_{H}\left(f_{t}\right)\right)} .
$$

Thus, the delay $\hat{\tau}\left(f_{t}\right)$, which maximizes the energy of $S\left(t, f_{t}, \tau\right)$, is

$$
\hat{\tau}\left(f_{t}\right)=\arg \left(\max _{\tau}\left|W\left(f_{t}\right) e^{2 \pi t f_{t}} \sum_{n=0}^{N-1} e^{j n\left(2 \pi \tau f_{t}+\phi_{H}\left(f_{t}\right)\right)}\right|\right)_{(17)} .
$$

This is equivalent to

$$
\hat{\tau}\left(f_{t}\right)=\arg \left(\max _{\tau}\left|\sum_{n=0}^{N-1} e^{j n\left(2 \pi \tau f_{t}+\phi_{H}\left(f_{t}\right)\right)}\right|\right)
$$

which leads to

$$
\phi_{H}\left(f_{t}\right)=-2 \pi \hat{\tau}\left(f_{t}\right) f_{t}[2 \pi] .
$$

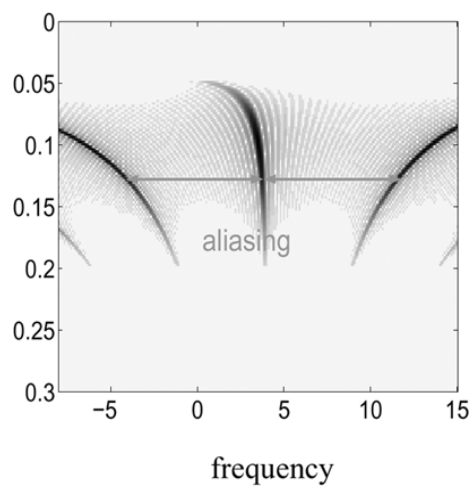

Fig. 4. Delay-frequency representation.

Applying a similar procedure to all $f_{t}$ s leads to a $\left(f_{t}, \tau\right)$ representation (referred to as a slant stack representation [10]). Note that this representation is actually the Fourier transform of the Radon transform [15]. Its modulus image is shown in Fig. 4; at the point of maximum energy for each line (i.e., each frequency), one can read $\phi_{H}\left(f_{t}\right)=2 \pi \hat{\tau}\left(f_{t}\right) f_{t}[2 \pi]$. Note that this time aliasing depends on frequency (see Fig. 4).

These two classical multisensor methods are fast and result in good estimations of the phase velocity between the sensors $\left(\phi_{H}\left(f_{t}\right)\right)$. In fact, these are the most widely used methods when dealing with large profiles (i.e., many traces). In addition, in the presence of several propagating waves where the frequency patterns of the different waves do not overlap, the analysis can be applied to each pattern independently because Fourier and Radon transforms are linear.

In practice, the main issue with these methods is trying to guarantee the condition of spatial stationarity (which means that $\phi_{H}$ is constant along all the different sensors) because the dispersion depends on the state of the ground, which may vary between the sensor array. This issue can be solved by processing the profile with a sliding window selecting only a few traces [16]. However, the consequence of applying these methods to only a few traces is that the spatial frequency resolution, which is inversely proportional to the number of used sensors $\left(\Delta f_{n}=1 / N\right)$ may be low. Thus, when we have several waves with similar frequency locations and velocities, overlapping phenomena become a problem. To illustrate this phenomenon, we consider a synthetic profile with four propagating waves [see Fig. 5(a)]. Two are nondispersive waves with the same negative velocity, and two are dispersive waves with positive velocity. When the number of sensors is large (50 sensors), the $f-k$ image [Fig. 5(b)] and slant stack image [Fig. 6(a)] are still useful, even if the different wave patterns overlap in the image. However, when the number of sensors drops to ten [keeping traces from 31 to 40; see Fig. 5(c)], the corresponding velocity-frequency representations [see Figs. 5(d) and 6(b)] are no longer useful.

To summarize, the two classical methods for performing dispersion estimation are based either on single-signal time-frequency or on multisignal velocity-frequency representations. We have outlined the advantages and drawbacks of these methods using a synthetic example. In the next section, we present a new algorithm, which is a combination of these two 


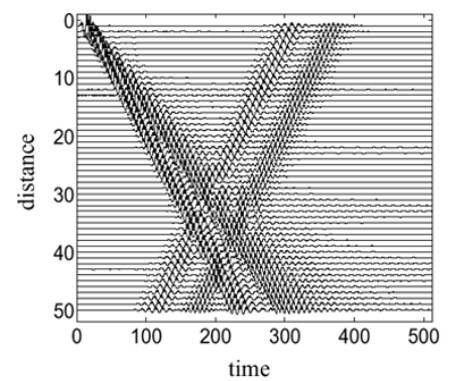

(a)

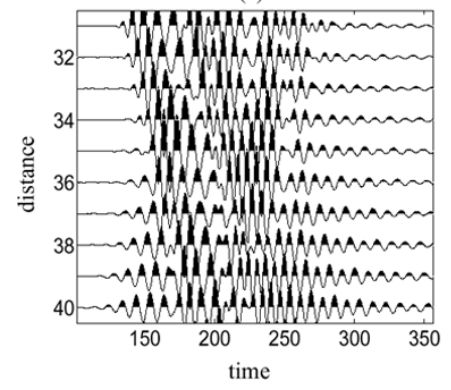

(c)

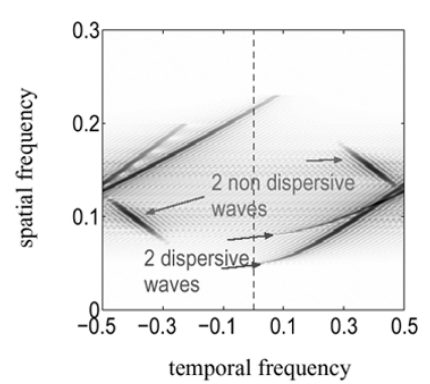

(b)

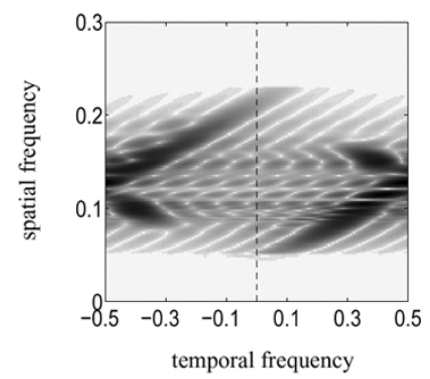

(d)
Fig. 5. Examples of spatial resolution issues in the $f-k$ representation. (a) Profile with 50 traces. (b) $|2 D F T|$ of (a). (c) Profile with ten traces. $|2 D F T|$ of (c).

classical methods. Its application is also illustrated using the same synthetic profile.

\section{Proposed Method}

\section{A. Time Resolution}

As we described in the previous section, in presence of several waves, the standard 1-D method uses the time variable in order to separate the patterns of the different waves, whereas the standard multisignal method uses the velocity variable to separate the patterns of the different waves. Basically, these methods are complementary. The method we propose will make use of both the time and velocity variables to separate the different wave patterns. There are two ways of presenting our method. On the one hand, it can be considered to be an $f-k$ spectrum representation, where time-resolution is introduced. As we will see in this section, the introduction of time resolution is done by analyzing the profile around each temporal frequency $f_{t}$ with a short time duration sine wave instead of the complex exponential $e^{-2 \pi j f_{t} t}$. On the other hand, the method can also be seen as the construction of a TFR, where the energy of waves which propagate at a selected velocity are amplified. This will be highlighted in the next section.

This section will be presented in a manner similar to that of the previous one, where multisignal analysis was discussed. The main difference to note is that when one analyzes the profile at the temporal frequency $f_{t}$ to estimate $\phi_{H}\left(f_{t}\right)$, we convolve the profile with a short time duration sine wave localized around $f_{t}$ noted $\psi_{f_{t}}$ instead of convolving it with the complex exponential $e^{-2 \pi j f_{t} t}$. This wavelet $\Psi_{f_{t}}(f)\left(\right.$ where $\left.\Psi_{f_{t}}(f)=F T_{t}\left[\psi_{f_{t}}(t)\right]\right)$ is a bandpass filter. In this paper, we use the Morlet wavelet,

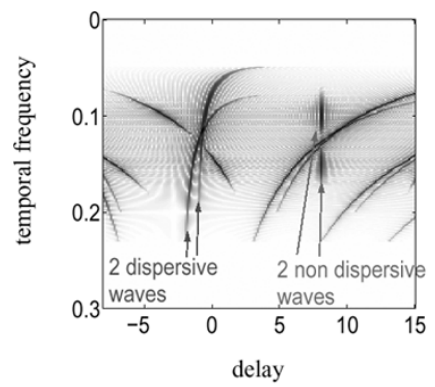

(a)

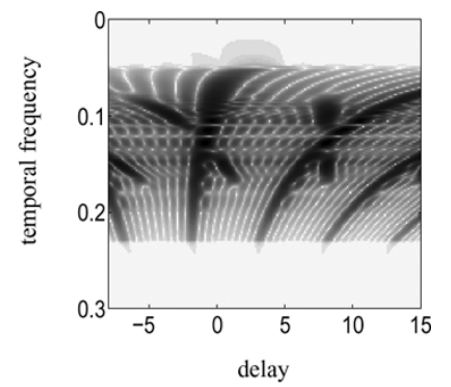

(b)
Fig. 6. Examples of spatial resolution issues in the slant-stack representation. (a) Profile with 50 traces. (b) Profile with ten traces.

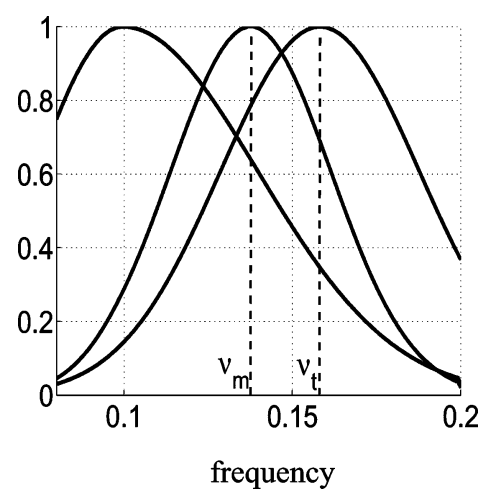

Fig. 7. Example of the frequency resolution issue. The amplitude maxima of the curves are normalized to 1 . On the left is the $\mathrm{FT}_{t}$ modulus of the profile $p$, on the right is the $\mathrm{FT}_{t}$ modulus of the wavelet $\psi$, and in the middle is the $\mathrm{FT}_{t}$ modulus of the filtered profile $p_{\psi_{f_{t}}}$.

which is a sine wave with a Gaussian envelope. The filtered profile $p_{\psi_{f_{t}}}$ can be written as

$$
p_{\psi_{f_{t}}}(n, t)=F T^{-1}\left[W(f) \Psi_{f_{t}}(f) e^{j n \phi_{H}(f)}\right](t) .
$$

The filters applied are local in time. The increase in time resolution and the consequently decreased frequency resolution are shown by comparing Figs. 3(c) and 8(c), and Figs. 3(d) and $8(\mathrm{~d})$. One of the problems with low-frequency resolution is that the product of the wave spectrum $W(f)$ and the wavelet spectrum $\Psi_{f_{t}}(f)$ is not necessarily centered at $f_{t}$ (see Fig. 7). This means that when we find the correction that aligns the wavelets in Fig. 8(c) and moves the pattern on the spatial frequency axes (vertical) in Fig. 8(d), we estimate the correction that compensates the dispersion at $f=f_{m}$ and not $f=f_{t}$. Our final goal is to estimate the function $f_{t} \mapsto \phi_{H}\left(f_{t}\right)$. To address this issue, the main frequency $f_{m}$ is estimated using the instantaneous frequency of the filtered signal [17] (we will return to this point after having described the proposed algorithm in Section III-B). When several estimations are available, the function $f_{t} \mapsto \phi_{H}\left(f_{t}\right)$ is estimated by extrapolating the function $f_{m} \mapsto \phi_{H}\left(f_{m}\right)$.

Assuming that the function $f \mapsto \phi_{H}(f)$ can be differentiated twice, it is possible to consider a wavelet whose bandwidth is small enough compared with the derivative of the curvature of the ridge in Fig. 8(b) so that we can assume that the pattern in 


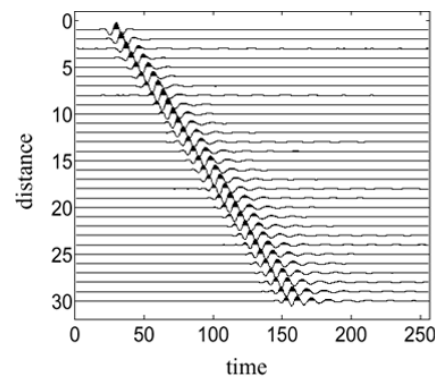

(a)

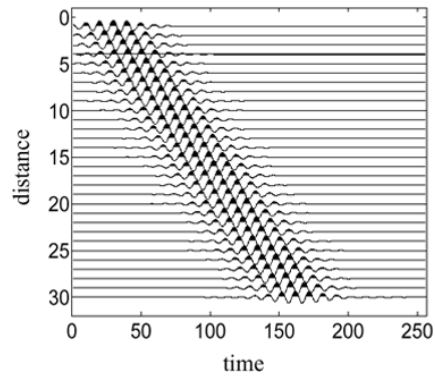

(c)

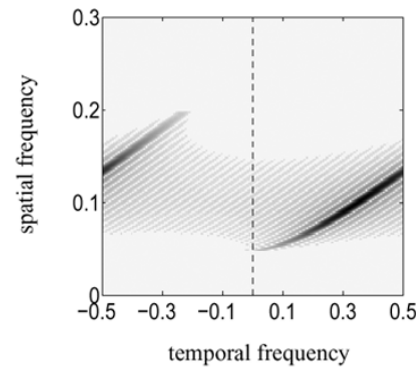

(b)

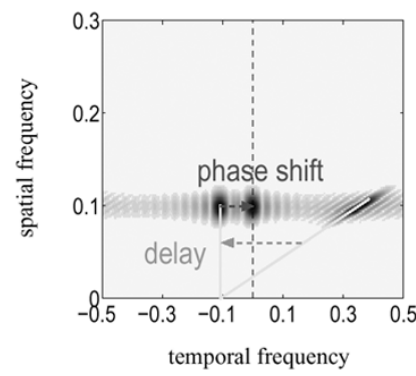

(d)
Fig. 8. Profile filtered by the wavelet. (a) Initial profile in time. (b) $|2 D F T|$ of (a). (c) Profile filtered by the wavelet. (d) $|2 D F T|$ of (c).

Fig. 8(d) is a linear segment. This is equivalent to assuming that the phase of the propagation filter can be approximated around $f_{m}$, to the first order, by

$$
\phi_{H}(f)=\phi_{H}\left(f_{m}\right)+\phi_{H}^{\prime}\left(f_{m}\right)\left(f-f_{m}\right) .
$$

By defining $\phi_{0}\left(f_{m}\right)=\phi_{H}\left(f_{m}\right)-\phi_{H}^{\prime}\left(f_{m}\right) f_{m}$ and $2 \pi \tau_{s}\left(f_{m}\right)=$ $-\phi_{H}^{\prime}\left(f_{m}\right)$, this can be rewritten as ${ }^{3}$

$$
\phi_{H}(f)=\phi_{0}\left(f_{m}\right)-2 \pi f \tau_{s}\left(f_{m}\right) .
$$

Substituting (22) into (20) leads to

$p_{\psi_{f_{t}}}(n, t)=F T^{-1}\left[W(f) \Psi_{f_{t}}(f) e^{j n\left(\phi_{0}\left(f_{m}\right)-2 \pi f \tau_{s}\left(f_{m}\right)\right)}\right]_{(}(t)$

which is equal to

$$
p_{\psi_{f_{t}}}(n, t)=w(t) * \psi_{f_{t}}(t) * \delta\left(t-n \tau_{s}\left(f_{m}\right)\right) \cdot e^{j n \phi_{0}\left(f_{m}\right)}
$$

This means that the propagation of $p_{\psi_{f_{t}}}$ can be approximated by the phase shift $\phi_{0}\left(f_{m}\right)$ followed by the delay $\tau_{s}\left(f_{m}\right)$. This is in agreement with the observation in Fig. 8(c). Thus, the dispersion at $f_{m}$ can be compensated by inserting a phase shift $\phi$ and a delay $\tau$ [symbolized by both arrows in Fig. 8(d)].

In order to estimate the correction (phase shift + delay) that compensates for the dispersion, we consider the summation of all traces after correction:

$$
\begin{aligned}
& S\left(t, f_{t}, \phi, \tau\right) \\
& =\sum_{n=0}^{N-1} w(t) * \psi_{f_{t}}(t) * \delta\left(t+n\left(\tau-\tau_{s}\left(f_{m}\right)\right)\right) \cdot e^{j n\left(\phi_{0}\left(f_{m}\right)+\phi\right)}
\end{aligned}
$$

${ }^{3}$ Here, $\tau_{s}\left(f_{m}\right)$ is the delay between two sensors and not between the sensor and the source. like in Section II-A.

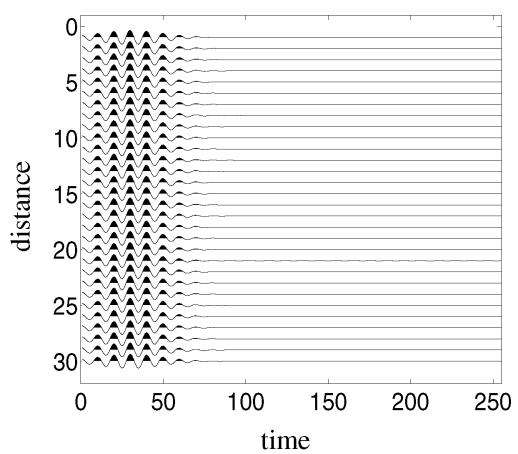

(a)

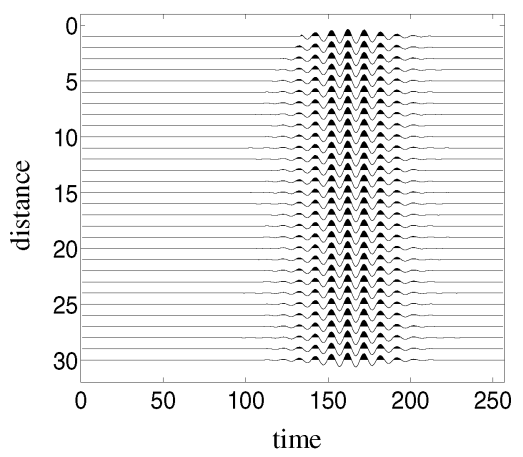

(b)

Fig. 9. Propagation compensation of the filtered profile $p_{\psi_{f_{t}}}$ on (a) the first sensor (a) and on (b) the last sensor.

which becomes

$S\left(t, f_{t}, \phi, \tau\right)$

$=w(t) * \psi_{f_{t}}(t) * \sum_{n=0}^{N-1} \delta\left(t+n\left(\tau-\tau_{s}\left(f_{m}\right)\right)\right) \cdot e^{j n\left(\phi_{0}\left(f_{m}\right)+\phi\right)}$.

It is clear that the dispersion is compensated when $\left(\sum_{n=0}^{N-1} \delta\left(t+n\left(\hat{\tau}-\tau_{s}\left(f_{m}\right)\right)\right) \cdot e^{j n\left(\phi_{0}\left(f_{m}\right)+\hat{\phi}\right)}=N \delta(t)\right)$. When this occurs [see Fig. 9(a)], the sum $S$ is localized in time because $\psi_{f_{t}}$ and $w$ are both localized in time. In addition, if we choose $\psi_{f_{t}}$ to be an analytic symmetric wavelet, the maximum magnitude of $S$ is located on the arrival time of the wave (at the frequency $f_{m}$ ) on the first trace.

In practice, the waves, when several are present, usually leave at the same instant from the source. Thus, they have more chance to be separated in time when arriving at the last sensor. Therefore, instead of compensating the propagation to get the arrival time of the wave on the first sensor, we reverse the distance axis. In this way, when the propagation is compensated, one gets Fig. 9(b), and the maximum magnitude of $S\left(t, f_{t}, \phi, \tau\right)$ is located at the arrival time of the wave on the last sensor (noted $\left.t_{a}\left(f_{m}\right)\right)$

In the standard multisignal method, we apply one correction (phase shift or delay), and we store the energy of the signal corresponding to the sum of all traces. In our method, we apply two corrections (phase shift and delay), but we actually want a third piece of information: the arrival time of the wave $t_{a}\left(f_{m}\right)$ on the last sensor. To estimate $t_{a}\left(f_{m}\right)$, we propose for each arrival time $t$ on the last sensor to look for the maximum magnitude on the 
signal corresponding to the sum of all traces among all double corrections (phase shifts and delays):

$$
A_{f_{t}}(t)=\left|S\left(t, f_{t}, \hat{\phi}\left(t, f_{t}\right), \hat{\tau}\left(t, f_{t}\right)\right)\right|
$$

where $\left(\hat{\phi}\left(t, f_{t}\right), \hat{\tau}\left(t, f_{t}\right)\right)=\arg \max _{(\tau, \phi)}\left|S\left(t, f_{t}, \phi, \tau\right)\right|$.

The advantage is that in presence of several waves with different arrival times $A_{f_{t}}(t)$ has several local maxima localized at the different arrival times. Furthermore, in order to concentrate the patterns around the local maxima of $A_{f_{t}}(t)$, when estimating the maximum of magnitude of $S\left(t, f_{t}, \phi, \tau\right)$, we only consider the corrections $(\tau, \phi)$ for which $t \mapsto\left|S\left(t, f_{t}, \phi, \tau\right)\right|$ has a local maximum at $t$ :

$$
\left(\hat{\phi}\left(t_{a}, f_{t}\right), \hat{\tau}\left(t_{a}, f_{t}\right)\right)=\arg \max _{(\tau, \phi) \in I_{t_{a}}}\left|S\left(t_{a}, f_{t}, \phi, \tau\right)\right|
$$

where $I_{t_{a}}$ is the set of all pairs $(\tau, \phi)$ such that $t \mapsto$ $\left|S\left(t, f_{t}, \phi, \tau\right)\right|$ has a local maximum at $t_{a}$.

At this step, the frequency $f_{m}$ is still unknown. It must be estimated for each double correction using the instantaneous frequency

$$
f_{i}\left(t, f_{t}, \phi, \tau\right)=\frac{1}{2 \pi} \frac{d \phi_{S}\left(t, f_{t}, \phi, \tau\right)}{d t}
$$

where $\phi_{S}\left(t, f_{t}, \phi, \tau\right)$ is the phase of $S\left(t, f_{t}, \phi, \tau\right)$.

Then, for each arrival time $t$, an estimation of the phase $\phi_{H}\left(f_{m}\right)$ is given using (22):

$$
\hat{\phi}_{H}\left(t, f_{t}\right)=-\hat{\phi}\left(t, f_{t}\right)-2 \pi f_{i}\left(t, f_{t}\right) \hat{\tau}\left(t, f_{t}\right)[2 \pi] .
$$

In our example with one wave, the magnitude function $A_{f_{t}}$ results in one maximum localized at the arrival time of the wave $t_{a}$ on the last sensor and at this sample $\hat{\phi}_{H}\left(t_{a}, f_{t}\right)=\phi_{H}\left(f_{m}\right)$.

\section{B. Application to All Frequencies}

The previous paragraph details how our algorithm performs the estimation of $\phi_{H}$ at a specific frequency $f_{m}$. Applying a similar procedure to each frequency means that we apply a filterbank $\Psi_{f_{t}}(f)$ to the profile and then, for each filter output, look for the correction that maximizes the magnitude of the sum of all traces. As the filtering operation is linear, we can apply the filterbank after the correction and the summation of the traces and, thus, estimate the best correction for all the frequencies simultaneously. This has the advantage of greatly reducing the cost of calculation because the set of corrections are applied only once. The application of this operation means that for each delay and phase shift correction, we apply a linear TFR to the signal corresponding to the summation of all traces to visualize where in the time-frequency plane the summation amplifies the energy. Stacking the different TFRs deduced from the different corrections leads to a volume that can be interpreted as a time-frequency-velocity volume. However, in order to visualize all this information, we choose to project this three-dimensional (3-D) information onto the time-frequency plane: For each (time, frequency) location, we look for the maximum magnitude among

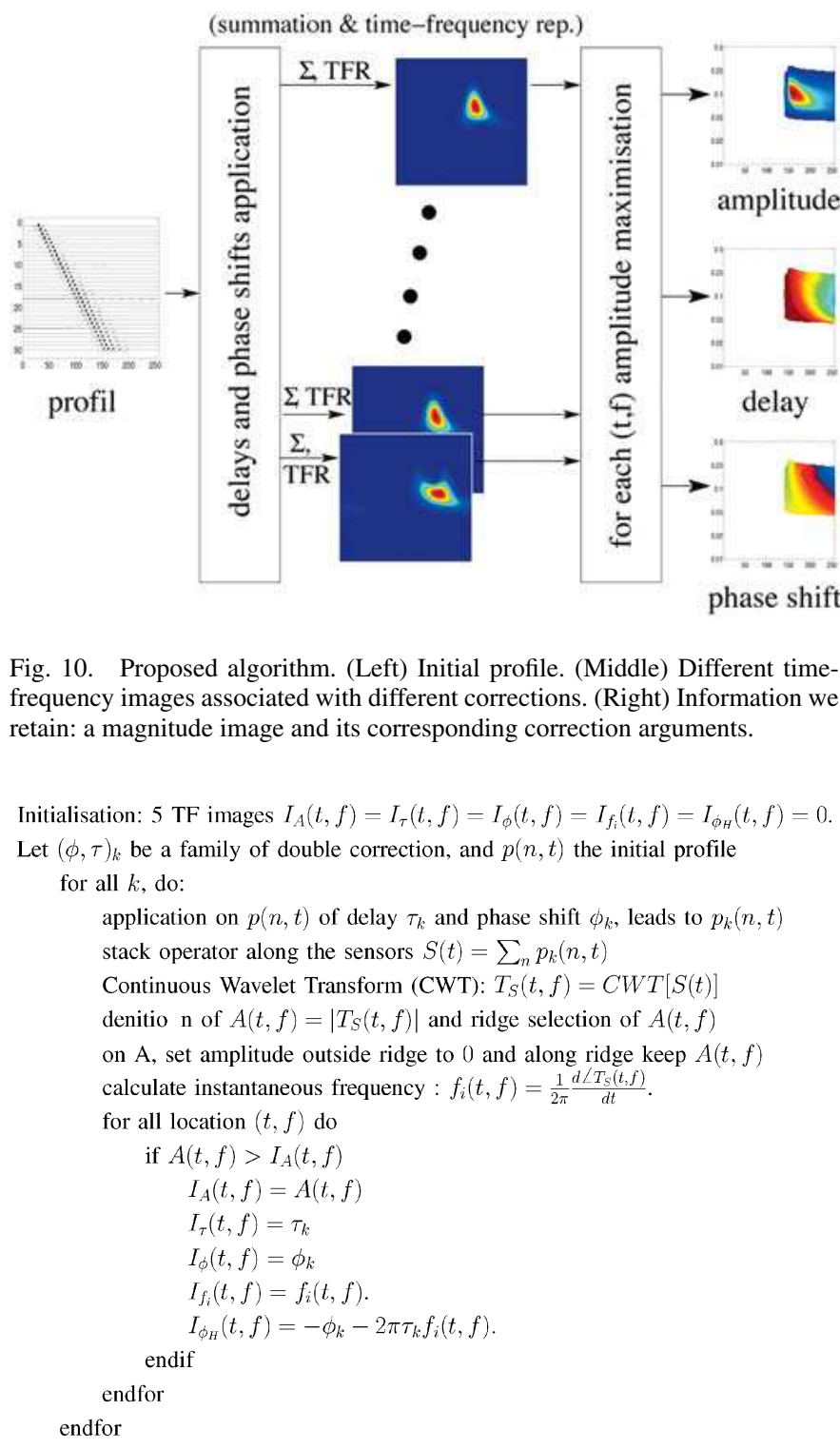

Fig. 11. Algorithm to generate five time-frequency images.

all the time-frequency images. This is illustrated in Fig. 10, where on the right, we obtain the magnitude image and both of the corresponding argument correction images: delay and phase shift, respectively. Note that in order to simplify the legibility of the data, we plot all the pixels whose value on the magnitude image is smaller than $1 \%$ of the maximum of the image in white.

In order to have a good TFR, the linear TFR we choose is the continuous wavelet transform (CWT) [13], [18]. In addition, the mother wavelet should be symmetric, analytic, and have a good TFR that is similar to seismic waves. For these reasons, we choose the Morlet wavelet [19].

The resulting algorithm is detailed in Fig. 11. On completion of the algorithm, we obtain five images in the time-frequency plane, giving, respectively, the magnitude $I_{A}(t, f)$, the delay $I_{\tau}(t, f)$, the phase shift $I_{\phi}(t, f)$, the frequency $I_{f_{i}}(t, f)$ and the dispersion phase estimators $I_{\phi_{H}}(t, f)$. Four of these images are shown in our synthetic example (see Fig. 12). The image of $I_{f_{i}}(t, f)$ is not shown because it has no visual interest. Note that the magnitude image is very similar to the TFR of the wave 


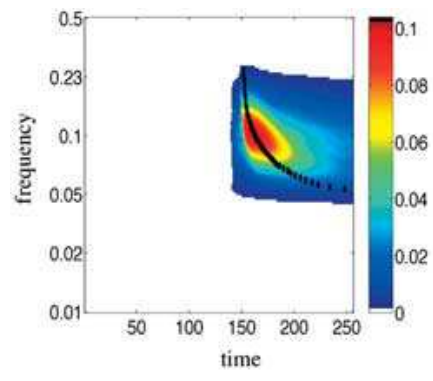

(a)

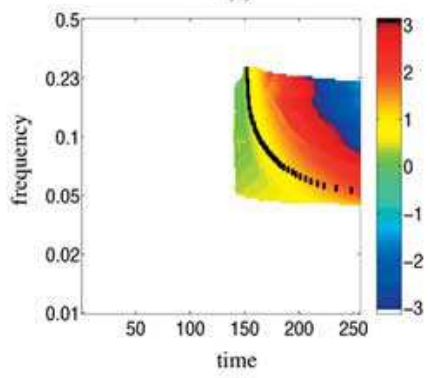

(c)

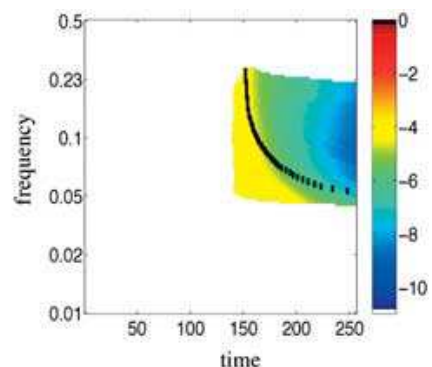

(b)

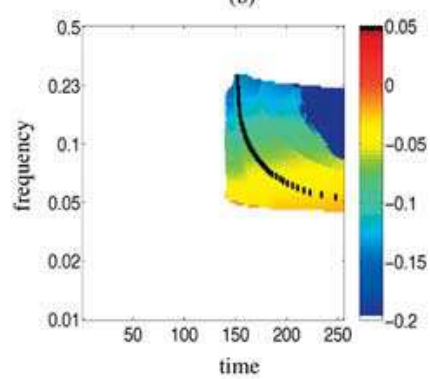

(d)

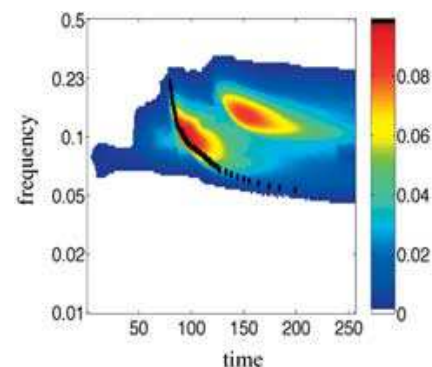

(a)

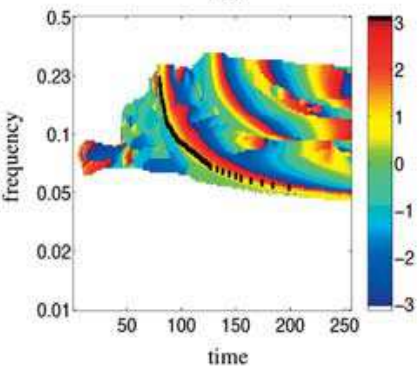

(c)

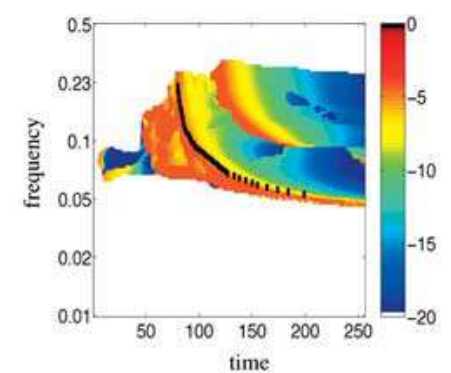

(b)

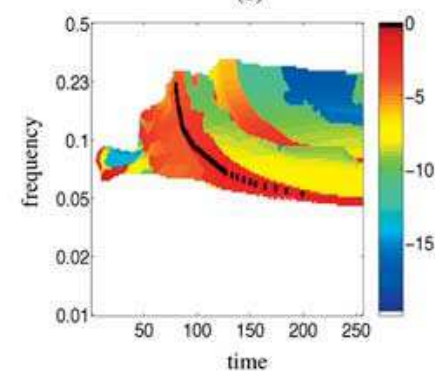

(d)

Fig. 12. Description of the data by four time-frequency images. (a) Magnitude image $I_{A}(t, f)$. (b) Delay image $I_{\tau}(t, f)$. (c) Phase shift image $I_{\phi}(t, f)$. (d) Phase image $I_{\phi_{H}}(t, f)$.

on the last sensor. The spreading of the pattern in time is proportional to the number of sensors present. In order to obtain the narrowest pattern, one should use only two sensors. However, increasing the number of sensors is sometimes necessary when noise is present in the data. Usually, one knows if there is some noise in the data by looking at the time-frequency patterns of the amplitude image. This noise can either be random or another wave field. Following this, the pattern on the magnitude image is used to estimate a new ridge. This is shown in black on the four images. Then, from the location corresponding to this ridge, the delay $\tau_{s}(f)$ and the phase $\phi_{H}(f)$ can be read from Fig. 12(b) and (d), respectively. Their corresponding frequency $f_{m}$ is read from the image of $I_{f_{i}}(t, f)$. Finally, by extrapolating the curve $f_{m} \mapsto \phi_{H}\left(f_{m}\right)$ and $f_{m} \mapsto \tau_{s}\left(f_{m}\right)$, one can simultaneously estimate the dispersion phase and group delay between the sensors.

Note that this algorithm could be more efficient if some $a$ priori knowledge of the wave's velocity is available. For example, if after analysis with the classical Fourier methods we know the approximate wave velocity, we can insert a test line into the algorithm (see Fig. 11) to remove some unwanted cases.

Now, we apply our algorithm to the previous synthetic example (see Fig. 5), where four waves are present within the profile (composed of ten sensors). We use the a priori information that the dispersive waves in which we are interested have a positive velocity. Therefore, only positive delay corrections $\tau_{k}$ have be applied. The propagations of nondispersive waves have yet to be compensated. Thus, when we estimate the maximum magnitude among all corrections, only the dispersive waves are present. In addition, thanks to the time resolution (see the resulting Fig. 13), both dispersive waves are separated, and they do not overlap in the time-frequency plane. In this example, our algorithm manages to separate the waves, whereas classical

Fig. 13. Four time-frequency images showing both dispersive waves. (a) Magnitude image $I_{A}(t, f)$. (b) Delay image $I_{\tau}(t, f)$. (c) Phase shift image $I_{\phi}(t, f)$. (d) Phase image $I_{\phi_{H}}(t, f)$.

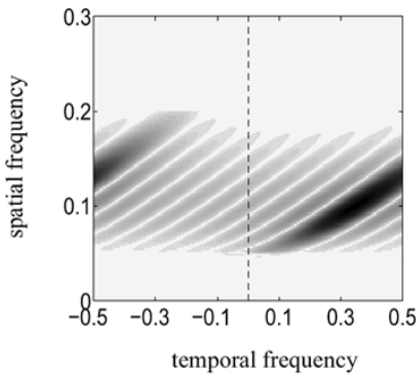

(a)

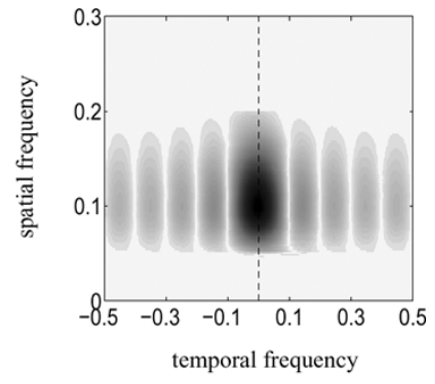

(b)
Fig. 14. Dispersion estimation result for a dispersive wave. (a) $|2 D F T|$ of the dispersive wave. (b) $|2 D F T|$ after correction.

methods do not (see Figs. 2 and 5). Finally, for both dispersive waves, the group delay $\tau_{s}(f)$ and the phase $\phi_{H}(f)$ can be read from images (b) and (d) of Fig. 13 at the position corresponding to the ridge in the pattern of the corresponding wave (one of these ridges is shown in black in Fig. 13). In order to check our results for the synthetic wave, we plot, in Fig. 14, the $f-k$ spectra before and after correction for the dispersion of the wave. The image shows that the dispersion has been perfectly compensated for and has been correctly estimated.

The presented algorithm is related to both velocity- and timefrequency representations but is more robust since it can use time and velocity variables to separate the different waves. Our method actually constructs a TFR of the waves as they arrive at the last sensor and, to do it, simultaneously uses the information in all the traces.

\section{Computation Cost Discussion and Parameter Settings}

From a computational point of view, our method is not competitive with the standard methods since we need to estimate two corrections (phase shift and delay) instead of one. Applying our 


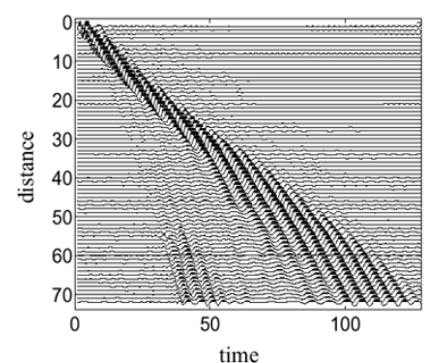

(a)

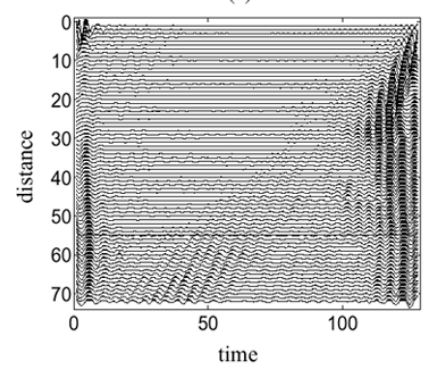

(c)

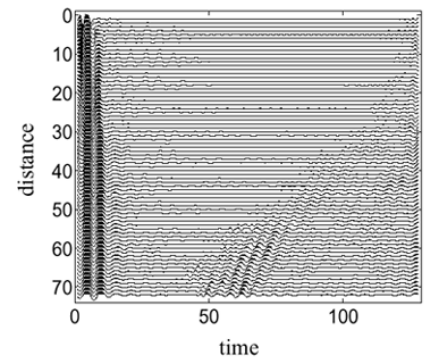

(e)

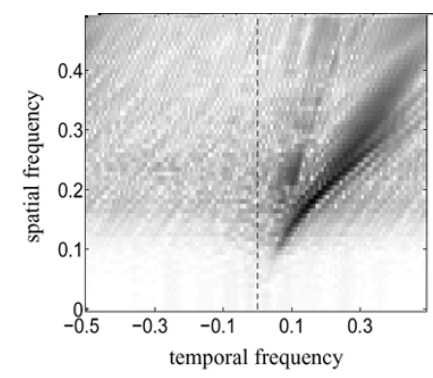

(b)

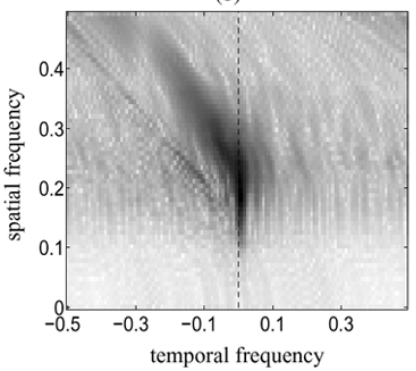

(d)

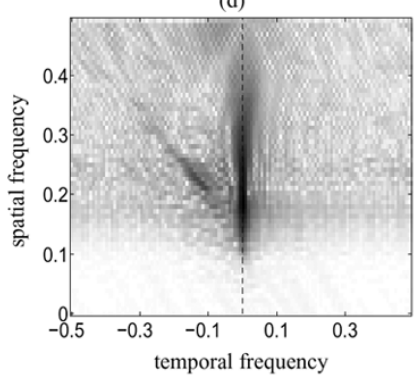

(f)
Fig. 15. Love wave dispersion correction. (a) Initial profile. (b) $|2 D F T|$ of (a). Results using (c) and (d) the standard Fourier method and (e) and (f) the proposed method.

method to large profiles will require to introduce optimization tools because what takes time is the application of the entire correction family $(\phi, \tau)_{k}$.

Choosing this family, and thus affecting the calculation time, is the main parameter-setting issue. If we want to be very accurate in the estimation of $f \mapsto \phi_{H}(f)$, we need to try many corrections. In all the presented examples (synthetic and real), we used 0.1 samples as a delay step and $0.02 \mathrm{rad}$ as a phase shift step. The maxima and minima values of delay and phase are arbitrarily chosen.

\section{Results From ReAl Data SETS}

\section{A. First Real Data Set}

The first real data set we consider is shown in Fig. 15(a) and (b), in time-distance and in $f-k$ space, respectively. It contains a Love wave (surface wave). In the $f-k$ image, it appears that the dispersive wave's pattern is not perfectly defined; a high-frequency component of the pattern seems to be separated from the main one. This is due to substrate variations between the sensors. When the dispersion is estimated for the whole profile using velocity-frequency representations [1], it is not possible to align the high-frequency part of the image [see Fig. 15(c) and (d)]. When we apply our algorithm, we estimate the dispersion using a sliding window, which is two sensors wide, ensuring that
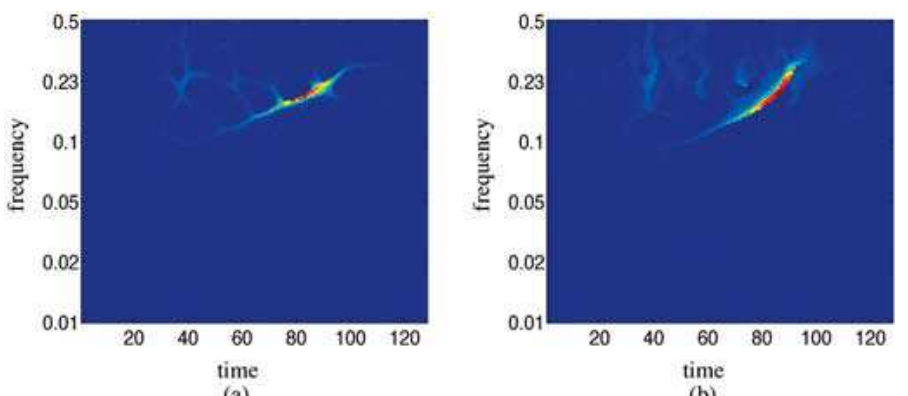

(a)

(b)

Fig. 16. Comparison between a reassigned scalogram and magnitude image for the presented algorithm. (a) Reassigned scalogram. (b) Magnitude image $I_{A}(t, f)$.
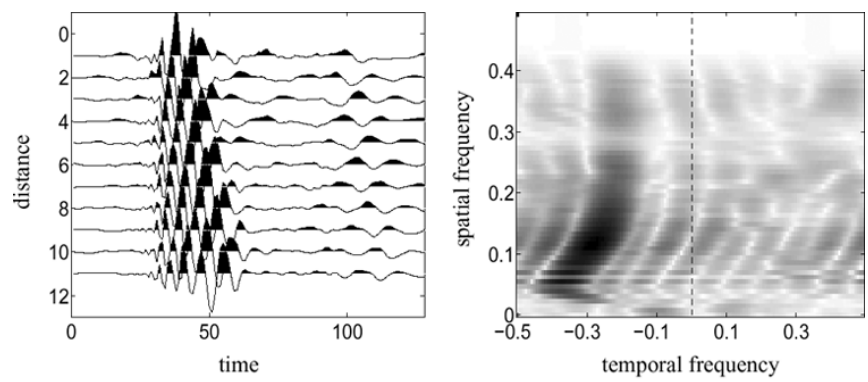

(a)
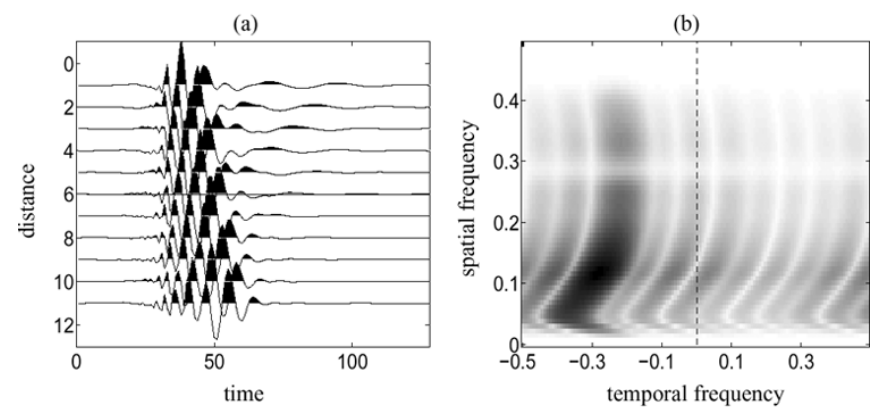

(c)

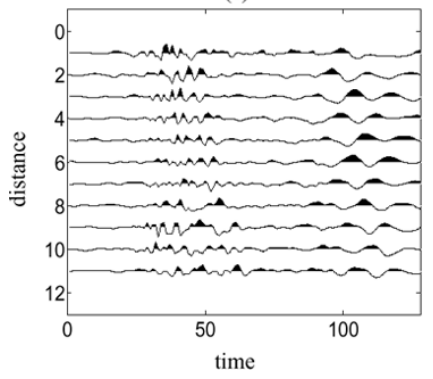

(e)

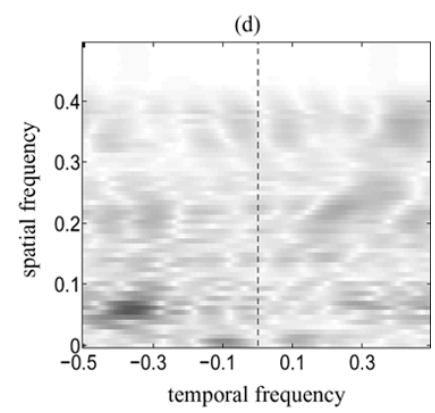

(f)

Fig. 17. Estimation and extraction of the energetic wave from the CGG data set. (a) Initial profile. (b) $|2 D F T|$ of (a). (c) Estimated profile of the wave. (d) $|2 D F T|$ of (c). (e) Profile after extraction. (f) $|2 D F T|$ of (e).

there are no problems due to propagation evolution between sensors. Fig. 15(e) and (f) shows the profile after dispersion correction using our proposed method. These plots clearly show that the dispersion is far more efficiently corrected than by using the standard method. The shape variation along the wave that still occurs in Fig. 15(e) is due to attenuation, which is not compensated. On the first few sensors, the high-frequency content of the wave has yet to disappear. This approximation does not disturb the phase estimation as it is based on phase alignment. This example demonstrates that our algorithm only needs two sensors to estimate the propagation between two sensors. 


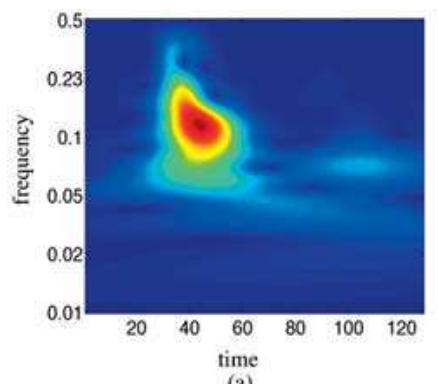

(a)

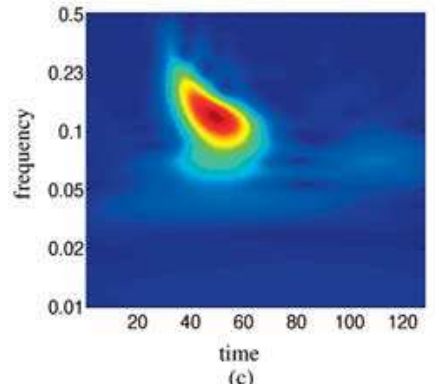

(c)

Fig. 18. Time-frequency description of two traces from the CGG data set. (a) Scalogram of trace 5. (b) Reassigned scalogram of trace 5. (c) Scalogram of trace 10. (d) Reassigned scalogram of trace 10 .

In comparison, the standard 1-D method is not able to find the phase $\phi_{H}$ between the sensors but only the group delay $\tau_{g}$ between the source and the sensor. Using (2), we can find the phase, but the estimation of the phase shift $\phi_{H}(0)$ requires extra processing. In order to compare the visual quality of the TFR resulting from our approach with that of the 1-D methods, the reassigned scalogram of a trace is shown in Fig. 16, as is the resulting magnitude image obtained using our algorithm. The results are similar, but the reassigned scalogram is noisier, and our method is more accurate. In addition, this also shows that the standard 1-D method is more sensitive to the noise than our method. As noise increases, the 1-D methods can no longer be used as their TFRs are no longer useful. Our proposed our method is still efficient as we can increase the size of the sliding window to stack the traces so that the noise becomes negligible.

\section{B. Second Real Data Set}

We now consider a second real data set supplied by the Compagnie Générale de Géophysique (CGG). The profile is shown in Fig. 17(a) in the time-distance domain. The $f-k$ space representation in Fig. 17(b) shows an energetic wave at the coordinates $(-0.3,0.12)$. The analysis of scalograms and reassigned scalograms in Fig. 18 enables us to observe an energetic wave [located at the coordinates $(40,0.12)$ ], as well as another pattern to its right at coordinates $(110,0.07)$. However, this is not perfectly clear, the images are noisy, and the ridge of the wave on the right does not enable to estimate its propagation.

Compared with magnitude images of the 1-D method, the time-frequency images given by our method presented in Fig. 19 are better. The magnitude images have less noise. In addition, the delay images show some coherence, which enables us to localize more easily the waves and provide additional information: its propagation. For instance, the wave located to the right of the point $(110,0.08)$ is clearly detectable in both traces.

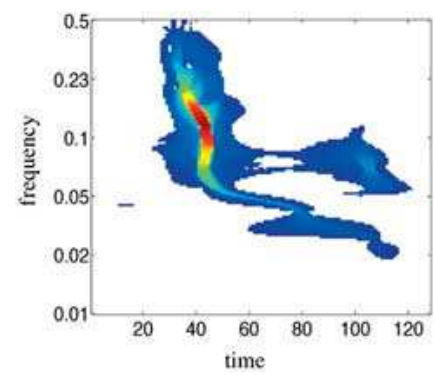

(a)

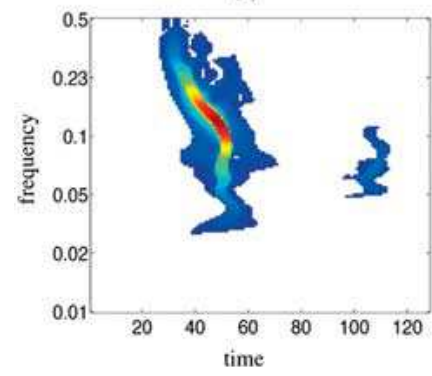

(c)

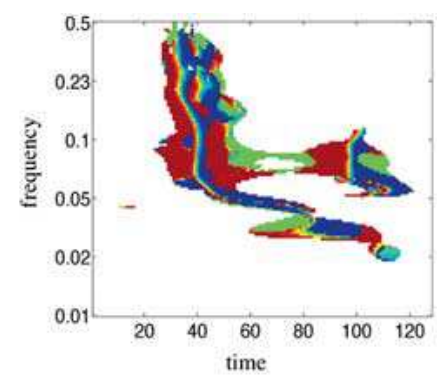

(b)

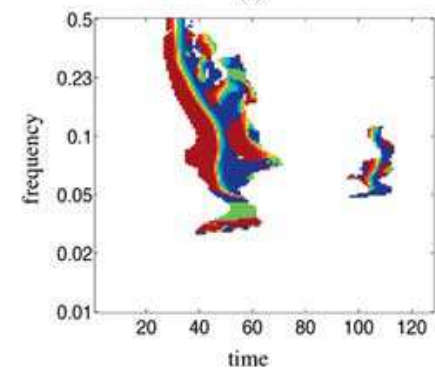

(d)
Fig. 19. Proposed description of the CGG data set. (a) Magnitude image $I_{A}(t, f)$ (trace 5). (b) Delay image $I_{\tau}(t, f)$ (trace 5). (c) Magnitude image $I_{A}(t, f)$ (trace 10). (d) Delay image $I_{\tau}(t, f)$ (trace 10).

We can ask why this wave does not appear in the $f-k$ image [see Fig. 17(b)]. To address this, we must estimate the energetic wave and then extract it from the profile [see Fig. 17(c) and (d)]. By looking at the residuals in Fig. 17(e) and (f), it appears that the wave on the right was not visible in the $f-k$ image because both wave patterns overlap at the point $(-0.35,0.08)$. This explains the presence of some oscillations in Fig. 17(b) at that point. We can conclude that $f-k$ image does not allow us to identify the wave on the right. This example serves to show us that $f-k$ images are not useful, whereas TFRs are, when waves have similar velocities but different arrival times.

\section{CONCLUSION}

A new algorithm aimed at estimating the wave dispersion from a linear array of sensors has been presented. The proposed method is based on the construction, from multisignal data, of a time-frequency representation of the propagating waves as they appear on the last sensor. The advantage is that we can select waves that propagate at a known velocity in this time-frequency image. Thus, this algorithm allows for efficient estimation, even when the different waves have similar velocities or nondisjoint time support. This is an advantage when compared with standard methods, which fail in either one of these situations.

However, from a practical point of view, it is important to underline that the application of our algorithm to large profiles would be far more computationally expensive than the use of classical methods. This is due to the fact that our method requires that we find two corrections instead of one, as per the classical ones. To reduce this problem, it will be necessary to use a priori information and optimize the calculation for the remaining unknown parameters. 


\section{ACKNOWLEDGMENT}

The authors wish to thank G. Armstrong for improving the quality of their use of the English language.

\section{REFERENCES}

[1] F. Glangeaud, J. L. Mari, J. Mars, and M. Nardin, "Dispersive seismic waves in geophysics," Eur. J. Environmental Eng. Geophys., vol. 3, pp. 265-306, 1999.

[2] F. Hlawatsch, A. Papandreou-Suppappola, and G. F. Boudreaux-Bartels, "The power classes - Quadratic time-frequency representations with scale covariance and dispersive time-shift covariance," IEEE Trans. Signal Process., vol. 47, no. 11, pp. 3067-3083, Nov. 1999.

[3] A. Papandreou-Suppappola, R. L. Murray, B.-F. Iem, and G. F. Boudreaux-Bartels, "Group delay shift covariant quadratic time-frequency representations," IEEE Trans. Signal Process., vol. 49, no. 11, pp. 2549-2563, Nov. 2001.

[4] J. L. Mari, F. Glangeaud, and F. Coppens, Signal Processing for Geologists and Geophysicists. Paris, France: Technip, 1999.

[5] A. Roueff, H. Pedersen, J. I. Mars, and J. Chanussot, "Simultaneous group and phase correction for the estimation of dispersive propagating waves in the time-frequency plane," in Int. Conf. Acoust., Speech, Signal Process., vol. 3, Hong Kong, 2003, pp. 429-432.

[6] A. Dziewonski, S. Bloch, and M. Landisman, "A technique for the analysis of transient seismic signals," Bull. Seismolog. Soc. Amer., vol. 59, no. 1, pp. 427-444, 1969.

[7] N. Shapiro and S. K. Singh, "A systematic error in estimating surfacewave group velocity dispersion curves and a procedure for its correction," Bull. Seismolog. Soc. Amer, vol. 89, pp. 1138-1142, 1999.

[8] H. A. Pedersen, J. I. Mars, and P. O. Amblard, "Improving group velocity measurements by energy reassignment," Geophys., vol. 68, no. 2, pp. 677-684, 2003.

[9] O. Yilmaz, Seismic Data Processing. Tulsa, OK: Soc. Exploration Geophys., 1987.

[10] G. A. McMechan and M. J. Yedlin, "Analysis of dispersive waves by wave field transformation,” Geophys., vol. 46, no. 6, pp. 869-874, 1981.

[11] R. G. Baraniuk, M. Coates, and P. Steeghs, "Hybrid linear/quadratic time-frequency attributes," IEEE Trans. Signal Process., vol. 49, no. 4, pp. 760-766, Apr. 2001.

[12] A. Francos and M. Porat, "Analysis and synthesis of multicomponent signals using positive time-frequency distribution," IEEE Trans. Signal Process., vol. 47, no. 2, pp. 493-504, Feb. 1999.

[13] P. Flandrin, Time-Frequency Distribution/Time-Scale Analysis. New York: Academic, 1999.

[14] F. Auger and P. Flandrin, "Improving the readability of time-frequency time-scale representation by the reassignment method," IEEE Trans. Signal Process., vol. 43, no. 5, pp. 1068-1089, May 1995.

[15] M. Dietrich, "Modeling of marine seismic profiles in the $t-x$ and $t-p$ domains," Geophys., vol. 53, no. 4, pp. 453-465, 1988.

[16] T. Stöcker, "Weyl filters for linear array data," IEEE Trans. Geosci.Remote Sens., vol. 41, no. 3, pp. 697-707, Mar. 2003.

[17] B. Boashash, "Estimating and interpreting the instantaneous frequency of a signal-Part 1: Fundamentals," Proc. IEEE, vol. 80, no. 4, pp. 519-538, Apr. 1992.

[18] A. Chakraborty and D. Okaya, "Frequency-time decomposition of seismic data using wavelet-based methods," Geophys., vol. 60, no. 6, pp. 1906-1916, 1995.

[19] L. Morlet, G. Arens, E. Fourgeau, and D. Giard, "Wave propagation and sampling theory, 1, complex signal and scattering in multilayered media," Geophys., vol. 47, no. 2, pp. 203-221, 1982.

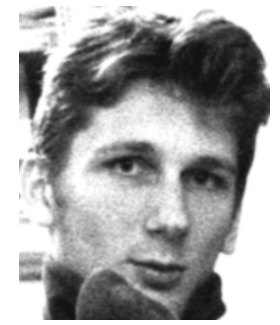

Antoine Roueff received the degree in electrical engineering in 2000 and the Ph.D. degree from the Laboratoire des Images et des Signaux (LIS), Grenoble National Polytechnic Institute (INPG), Grenoble, France, in 2003.

Since 2003, he has had a postdoctoral position with the Commissariat à l'Energie Atomique (CEA), Bruyères-le-Chatel, France. He is involved in the application of signal processing tools for the analysis of geophysical and underwater acoustical signal and image processing. His research interests include time-frequency and time-scale analysis, multicomponent and multisensor processing, and classification.

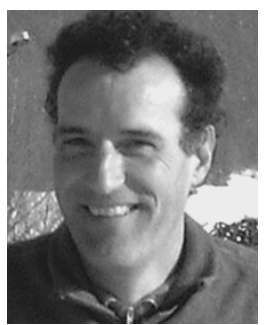

Jérôme I. Mars received the M.S. degree in geophysics in 1986 from Joseph Fourier University of Grenoble, Grenoble, France, and the Ph.D. degree in signal processing in 1988 from the Institut National Polytechnique of Grenoble.

From 1989 to 1992, he was a postdoctoral research with the Centre des Phénomènes Aléatoires et Geophysiques, Grenoble. From 1992 to 1995, he was a visiting lecturer and scientist at the Materials Sciences and Mineral Engineering Department, University of California, Berkeley. He is currently an Assistant Professor in signal processing with the Laboratoire des Images et des Signaux, Institut National Polytechnique de Grenoble, where he is the leader of the geophysical signal processing team. His research interests include seismic and acoustic signal processing, wavefield separation methods, time-frequency time-scale characterization, and applied geophysics.

Dr. Mars is a member of SEG and EAGE.

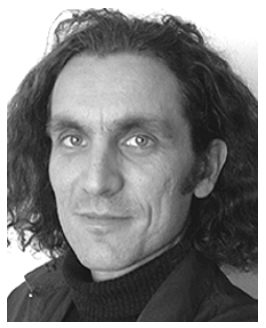

Jocelyn Chanussot received the electrical engineering degree from the Grenoble National Polytechnic Institute (INPG), Grenoble, France, in 1995. He then joined the Automatics and Industrial Micro-Computer Science Laboratory (LAMII), Savoie University, Annecy, France, from which he received the $\mathrm{Ph} . \mathrm{D}$. degree in 1998.

In 1999, he was with the Geography Imagery Perception laboratory (GIP), French National Defense Department (DGA, Délégation Générale de l'Armement). Since 1999, he has been an Associate Professor of signal and image processing with the Signals and Images Laboratory (LIS), INPG. He is involved with the team working on geophysical and underwater acoustical signal and image processing. His research interests include multicomponent image processing, nonlinear filtering, remote sensing, data fusion, and their applications to natural real bidimensional data (seismic data, sonar images).

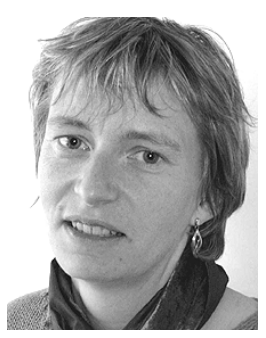

Helle Pedersen received the engineering degree in geophysics from l'Ecole de Physique du Globe, Strasburg, France, in 1990, the Masters degree from the University of Aarhus, Aarhus, Denmark, in 1991, and the Ph.D. degree from the University of Grenoble, Grenoble, France, in 1994.

Since 1995, she has been assistant professor in geophysics at the University of Grenoble. She specializes in seismic surface waves and seismic wave propagation in complex media. She is currently on sabbatical leave at the GeoforschungsZentrum Potsdam and University of Potsdam, Potsdam, Germany. 